\title{
Influence of Nephrologist Care on Management and Outcomes in Adults with Chronic Kidney Disease
}

\author{
Ana C. Ricardo, MD, MPH' , Jason A. Roy, PhD'², Kaixiang Tao, PhD'², Arnold Alper, MD, MPH , \\ Jing Chen, MD, MSC ${ }^{3}$, Paul E. Drawz, MD, MHS ${ }^{4}$, Jeffrey C. Fink, MD, MS $S^{5}$, Chi-yuan Hsu, MD, MSC , \\ John W. Kusek, $P h D^{7}$, Akinlolu Ojo, MD, PhD ${ }^{8}$, Martin Schreiber, $M D^{9}$, and Michael J. Fischer, \\ $M D, M S P H^{10,11}$ on behalf of the CRIC Study Investigators
}

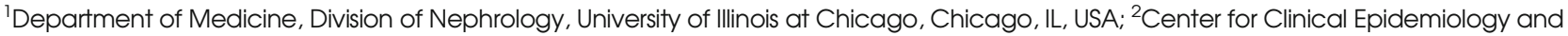
Biostatistics, University of Pennsylvania, Philadelphia, PA, USA; ${ }^{3}$ Department of Medicine, Tulane University, New Orleans, LA, USA; ${ }^{4}$ Department of Medicine, University of Minnesota, Minneapolis, MN, USA; ${ }^{5}$ Department of Medicine, University of Maryland, Baltimore, MD, USA; ${ }^{6}$ Department of Medicine, University of California, San Francisco, CA, USA; ${ }^{7}$ National Institute of Diabetes and Digestive and Kidney Diseases, National Institutes of Health, Bethesda, MD, USA; ${ }^{8}$ Department of Medicine, University of Michigan, Ann Arbor, MI, USA; ${ }^{\circ}$ Department of Nephrology and Hypertension, Cleveland Clinic Foundation, Cleveland, OH, USA; ${ }^{10}$ Center of Innovation for Complex Chronic Healthcare, Jesse Brown VA Medical Center,

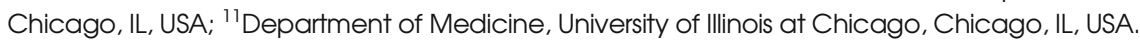

BACKGROUND: Predialysis nephrology care for adults with late stage chronic kidney disease (CKD) is associated with improved outcomes. Less is known about the effects of nephrology care in earlier stages of CKD.

OBJECTIVE: We aimed to evaluate the effect of nephrology care on management of CKD risk factors and complications, CKD progression, incident cardiovascular disease (CVD), and death.

DESIGN: This was a prospective cohort study.

PARTICIPANTS: Participants included 3855 men and women aged 21 to 74 years enrolled in the Chronic Renal Insufficiency Cohort (CRIC) Study with a mean (SD) estimated glomerular filtration rate (eGFR) at entry of 45 (17) $\mathrm{ml} / \mathrm{min} / 1.73 \mathrm{~m}^{2}$, followed for a median of 6.6 years.

MAIN MEASURES: The main predictor was self-reported prior contact with a nephrologist at study enrollment. Outcomes evaluated included CKD progression $(\geq 50 \%$ eGFR loss or end-stage renal disease), incident CVD, and death.

RESULTS: Two-thirds (67\%) of the participants reported prior contact with a nephrologist at study enrollment. They were younger, more likely to be male, non-Hispanic white, and had lower eGFR and higher urine protein ( $\mathrm{p}<$ 0.05). A subgroup with eGFR $30-<60 \mathrm{ml} / \mathrm{min} / 1.73 \mathrm{~m}^{2}$ and prior contact with a nephrologist were more likely to receive pharmacologic treatment for CKD-related complications and to report angiotensin-converting enzyme inhibitor or angiotensin receptor blocker (ACEi/ARB) use. After propensity score matching (for reporting prior contact with a nephrologist vs. not) and adjusting for demographic and clinical variables, prior contact with a

CRIC Study Investigators: Lawrence J. Appel, MD, MPH, Harold I. Feldman, MD, MSCE, Alan S. Go, MD, Jiang He, MD, PhD, John W. Kusek, PhD, James P. Lash, MD, Akinlolu Ojo, MD, PhD, Mahboob Rahman, MD

Electronic supplementary material The online version of this article (doi:10.1007/s11606-015-3452-x) contains supplementary material, which is available to authorized users.

Received October 21, 2014

Revised April 23, 2015

Accepted June 15, 2015

Published online July 3, 2015 nephrologist was not significantly associated with CKD progression, incident CVD or death ( $\mathrm{p}>0.05)$.

CONCLUSIONS: One-third of CRIC participants had not seen a nephrologist before enrollment, and this prior contact was subject to age, sex, and ethnic-related disparities. While prior nephrology care was associated with more frequent treatment of CKD complications and use of ACEi/ARB medications, there was neither an association between this care and achievement of guidelinerecommended intermediate measures, nor long-term adverse outcomes.

KEY WORDS: chronic kidney disease; nephrology care; outcomes.

J Gen Intern Med 31(1):22-9

DOI: $10.1007 / \mathrm{s} 11606-015-3452-\mathrm{x}$

(C) Society of General Internal Medicine 2015

\section{INTRODUCTION}

It is recommended that chronic kidney disease (CKD) patients receive nephrology care before dialysis initiation. Current guidelines recommend specialist referral for individuals with estimated glomerular filtration rate (eGFR) $<30 \mathrm{ml} / \mathrm{min} /$ $1.73 \mathrm{~m}^{2}$. 1,2 Absent or late predialysis nephrology care is associated with poorer blood pressure (BP) control, worse biochemical parameters, and lower receipt of permanent vascular access among patients at dialysis initiation. ${ }^{3,4}$ Furthermore, patients with CKD who are referred late to nephrologists (less than 3-4 months before initiation of dialysis) have been shown to have higher mortality in the first $\mathrm{t}^{3,5-7}$ or second $^{8,9}$ year after initiation of dialysis.

Less is known about the benefits of receiving nephrology care in earlier CKD stages. Several studies have shown that outpatient nephrology care may result in slowing kidney function decline and/or improving survival. ${ }^{10-14}$ However, these reports are limited by small sample sizes, lack of racial/ ethnicity diversity, retrospective design or short duration of follow-up. 
The purpose of this study was to characterize current patterns of nephrology care and to examine the effect of nephrology care on management of CKD risk factors and complications, CKD progression, incident cardiovascular disease (CVD), and death among Chronic Renal Insufficiency Cohort (CRIC) Study participants. We hypothesized that receipt of nephrology care will be associated with lower risk of CKD progression, CVD events and all-cause mortality, and that this association would in part be due to earlier implementation by a nephrologist of guideline-recommended therapies such as BP management.

\section{MATERIALS AND METHODS}

\section{Study Population}

The design, methods and baseline characteristics of CRIC participants have been previously published. ${ }^{15-17}$ The CRIC Study recruited 3939 adults from May 2003 to June 2008, aged 21-74 years, with mild to moderate CKD from general medicine and specialty clinics at seven clinical centers across the U.S. (Ann Arbor, Michigan; Baltimore, Maryland; Chicago, Illinois; Cleveland, Ohio; New Orleans, Louisiana; Philadelphia, Pennsylvania; and Oakland, California). Participants were followed with annual in-clinic visits and interim telephone contact. Exclusion criteria included inability to consent, institutionalization, pregnancy, and certain chronic conditions. ${ }^{15-17}$ The study protocol was approved by Institutional Review Boards of participating centers and is in accordance with the principles of the Declaration of Helsinki. All study participants provided written informed consent.

\section{Prior Contact with a Nephrologist}

Prior nephrology contact was determined at study entry by answering "yes" to the question: "Have you ever seen a nephrologist or kidney doctor?" In addition, we evaluated continuity of nephrology care among participants with a compelling indication for specialist referral (i.e., eGFR $<30 \mathrm{ml} / \mathrm{min} / 1.73 \mathrm{~m}^{2}$ or urine protein $\geq 3 \mathrm{~g} /$ day). ${ }^{1,2}$ To ascertain continuity of nephrology care, the following question was asked during each annual visit: "Since the last CRIC visit, did you see nephrologist/ kidney doctor or any other doctor/health professional(s) for your kidney problems?" Answering "yes" to that question triggered the following question: "Since the last CRIC visit, how many times did you see a nephrologist/kidney doctor for your kidney problems?" Taking into account reported visits to nephrologist/ kidney doctor (not primary care providers or other specialists), we classified continuity of nephrology care as follows: persons who reported nephrology contact at least once per year during follow-up (100\% continuity of nephrology care); participants who reported nephrology contact during most but not all follow-up visits (continuity of nephrology care 51-99 \%); and those who reported nephrology contact during less than half of the follow-up visits (continuity of nephrology care $\leq 50 \%$ ).

\section{Outcomes}

In cross-sectional analyses, prior nephrology contact at study entry was the outcome of interest. We also examined achievement of CKD management guideline measures at baseline, including BP level, laboratory parameters (bicarbonate, phosphate, parathyroid hormone and hemoglobin) and use of angiotensin converting enzyme inhibitors or angiotensin receptor blocker (ACEi/ARB). Because CRIC participants were recruited between 2003 and 2008, we used the goals set by the U.S. National Kidney Foundation Kidney Disease Outcomes Quality Initiative (NKF KDOQI) published between 2003 and 2006. ${ }^{18-20}$

For longitudinal analyses, we evaluated the following outcomes: 1) CKD progression [50\% eGFR decline from baseline, or end-stage renal disease (ESRD, initiation of dialysis therapy or kidney transplantation)]; 2) incident CVD (myocardial infarction, heart failure, stroke, or peripheral arterial disease) and 3) all-cause death. Ascertainment of time to eGFR halving was imputed assuming a linear decline in kidney function between in-person annual visit measures for time-toevent analyses. Ascertainment of ESRD was supplemented by cross-linkage of participants with the US Renal Data System. CVD events were adjudicated by physician reviewers. ${ }^{15}$ Deaths were ascertained from next of kin reports, death certificates, hospital records, and linkage with the Social Security Death Master File. Participants were followed up until the occurrence of death, voluntary withdrawal, or 31 March 2012.

\section{Covariates}

Sociodemographic characteristics (age, gender, race/ethnicity, annual household income, educational attainment, health insurance, smoking status), medical history and medications were self-reported at entry. BP and anthropometric measures were obtained using standard protocols. ${ }^{16}$ Hypertension was defined as systolic BP $\geq 140 \mathrm{mmHg}$, diastolic $\mathrm{BP} \geq 90 \mathrm{mmHg}$, or use of antihypertensive medications. History of CVD was defined as self-reported coronary heart disease (myocardial infarction or prior revascularization), heart failure, peripheral arterial disease, or stroke. Dyslipidemia was defined as fasting total cholesterol $\geq 240 \mathrm{mg} / \mathrm{dl}$, low density lipoprotein $\geq$ $160 \mathrm{mg} / \mathrm{dl}$, high-density lipoprotein $\leq 40 \mathrm{mg} / \mathrm{dl}$, triglycerides $\geq 200 \mathrm{mg} / \mathrm{dl}$, self-reported high cholesterol, or use of lipidlowering medication. Diabetes was defined as fasting glucose $\geq 126 \mathrm{mg} / \mathrm{dL}$, random glucose $\geq 200 \mathrm{mg} / \mathrm{dL}$, or use of insulin or anti-diabetic medication. GFR was estimated using a CRIC 
equation. $^{21}$ Proteinuria was measured from 24-hour urine collection at baseline.

\section{Statistical Analysis}

Descriptive statistics were summarized as mean (SD) for continuous variables and frequency (proportion) for categorical variables. For skewed data distributions, natural logarithmic transformations were conducted, and/or data were presented as median (interquartile intervals). Chi-squared and analysis of variance were used to compare categorical and continuous variables, respectively. Logistic regression was used in cross-sectional analyses to determine factors associated with prior nephrology contact adjusted for demographic and clinical factors chosen based on prior literature. ${ }^{22-24}$ In longitudinal analyses, Cox proportional hazards models were used to examine the association of prior nephrology contact and outcomes. For the outcomes of CKD progression and incident CVD, death was treated as a censoring event. To minimize potential bias and confounding introduced by nonrandom assignment of participants to prior nephrology contact, a matched propensity score approach was utilized. A propensity score for the probability of prior nephrology contact was calculated for each participant using a logistic regression model incorporating demographic and clinical characteristics listed in Table 2. We matched 925 participants who reported prior nephrology contact with the nearest propensity-matched participant who did not report such contact using a greedy algorithm with a pre-specified 0.02 caliper distance, which corresponds approximately to matched pairs that are within $1 \% \mathrm{SD}$ of the propensity score. The success of

Table 1 Demographic and Clinical Characteristics of Study Participants by Contact with a Nephrologist Prior to CRIC Baseline Visit

\begin{tabular}{|c|c|c|c|c|}
\hline \multirow[t]{2}{*}{ Characteristic } & \multirow[t]{2}{*}{ Overall } & \multicolumn{2}{|c|}{ Prior Contact with a Nephrologist } & \multirow[t]{2}{*}{$p$} \\
\hline & & Yes & No & \\
\hline No. of participants & 3855 & 2591 & 1264 & \\
\hline Age, y & $58.1(11.0)$ & $57.5(11.6)$ & $59.4(9.7)$ & $<0.001$ \\
\hline Male sex & $2117(54.9 \%)$ & $1515(58.5 \%)$ & $602(47.6 \%)$ & $<0.001$ \\
\hline \multicolumn{5}{|l|}{ Race/ethnicity } \\
\hline Non-Hispanic White & $1613(41.8 \%)$ & $1130(43.6 \%)$ & $483(38.2 \%)$ & \multirow[t]{4}{*}{$<0.001$} \\
\hline Non-Hispanic Black & $1604(41.6 \%)$ & $1100(42.5 \%)$ & $504(39.9 \%)$ & \\
\hline Hispanic & $487(12.6 \%)$ & $263(10.2 \%)$ & $224(17.7 \%)$ & \\
\hline Other & $151(3.9 \%)$ & $98(3.8 \%)$ & $53(4.2 \%)$ & \\
\hline \multicolumn{5}{|l|}{ Annual Household Income, \$ } \\
\hline$\leq 20,000$ & $1210(31.4 \%)$ & $800(30.9 \%)$ & $410(32.4 \%)$ & \multirow[t]{5}{*}{0.35} \\
\hline $20,001-50,000$ & $933(24.2 \%)$ & $642(24.8 \%)$ & $291(23.0 \%)$ & \\
\hline $50,000-100,000$ & $721(18.7 \%)$ & $490(18.9 \%)$ & $231(18.3 \%)$ & \\
\hline$>100,000$ & $391(10.1 \%)$ & $271(10.5 \%)$ & $120(9.5 \%)$ & \\
\hline No response & $600(15.6 \%)$ & $388(15.0 \%)$ & $212(16.8 \%)$ & \\
\hline \multicolumn{5}{|l|}{ Educational Attainment } \\
\hline$<$ High school & $802(20.8 \%)$ & $510(19.7 \%)$ & $292(23.1 \%)$ & \multirow{3}{*}{0.05} \\
\hline HS graduate/some college & $1841(47.8 \%)$ & $1251(48.3 \%)$ & $590(46.7 \%)$ & \\
\hline$\geq$ College graduate & $1211(31.4 \%)$ & $830(32.0 \%)$ & $381(30.2 \%)$ & \\
\hline \multicolumn{5}{|l|}{ Health Insurance } \\
\hline None & $267(7.8 \%)$ & $159(6.9 \%)$ & $108(9.6 \%)$ & \multirow[t]{6}{*}{$<0.0001$} \\
\hline Medicaid/Public Aid & $497(14.5 \%)$ & $352(15.3 \%)$ & $145(12.9 \%)$ & \\
\hline Any Medicare & $1185(34.6 \%)$ & $794(34.6 \%)$ & $391(34.8 \%)$ & \\
\hline VA/Military/Champus & $189(5.5 \%)$ & $136(5.9 \%)$ & $53(4.7 \%)$ & \\
\hline Private/Commercial & $573(16.7 \%)$ & $313(13.6 \%)$ & $260(23.2 \%)$ & \\
\hline Unknown/Incomplete Data & $710(20.8 \%)$ & $544(23.7 \%)$ & $166(14.8 \%)$ & \\
\hline Smoker ( $\geq 100$ cig/lifetime) & $2105(54.6 \%)$ & $1443(55.7 \%)$ & $662(52.4 \%)$ & 0.05 \\
\hline Systolic Blood Pressure, $\mathrm{mmHg}$ & $128.4(22.1)$ & $128.8(22.2)$ & $127.7(21.8)$ & 0.18 \\
\hline Hypertension & $3317(86.0 \%)$ & $2282(88.1 \%)$ & $1035(81.9 \%)$ & $<0.001$ \\
\hline Coronary heart disease $^{\mathrm{a}}$ & $838(21.7 \%)$ & $581(22.4 \%)$ & $257(20.3 \%)$ & 0.14 \\
\hline Congestive heart failure $^{\mathrm{a}}$ & $372(9.6 \%)$ & $260(10.0 \%)$ & $112(8.9 \%)$ & 0.25 \\
\hline PVD $^{\mathrm{a}}$ & $259(6.7 \%)$ & $202(7.8 \%)$ & $57(4.5 \%)$ & $<0.001$ \\
\hline Stroke & $381(9.9 \%)$ & $263(10.2 \%)$ & $118(9.3 \%)$ & 0.43 \\
\hline Any CVD & $1285(33.3 \%)$ & $900(34.7 \%)$ & $385(30.5 \%)$ & 0.008 \\
\hline Dyslipidemia & $3165(82.1 \%)$ & $2163(83.5 \%)$ & $1002(79.3 \%)$ & 0.001 \\
\hline Diabetes mellitus & $1863(48.3 \%)$ & $1236(47.7 \%)$ & $627(49.6 \%)$ & 0.27 \\
\hline Body mass index, $\mathrm{Kg} / \mathrm{m}^{2}$ & $32.1(7.8)$ & $31.8(7.7)$ & $32.5(8.0)$ & 0.008 \\
\hline $\mathrm{eGFR}, \mathrm{ml} / \mathrm{min} / 1.73 \mathrm{~m}^{2}$ & $44.9(16.8)$ & $40.9(14.9)$ & $53.1(17.6)$ & $<0.001$ \\
\hline \multicolumn{5}{|l|}{ eGFR Category } \\
\hline $15-<30$ & $792(20.5 \%)$ & $705(27.2 \%)$ & $87(6.9 \%)$ & \multirow[t]{6}{*}{$<0.001$} \\
\hline $30-<40$ & $891(23.1 \%)$ & $676(26.1 \%)$ & $215(17.0 \%)$ & \\
\hline $40-<50$ & $831(21.6 \%)$ & $547(21.1 \%)$ & $284(22.5 \%)$ & \\
\hline $50-<60$ & $653(16.9 \%)$ & $374(14.4 \%)$ & $279(22.1 \%)$ & \\
\hline$\geq 60$ & $688(17.8 \%)$ & $289(11.2 \%)$ & $399(31.6 \%)$ & \\
\hline Urine Protein $(\mathrm{g} / 24 \mathrm{hr})$ & $0.2(0.1-0.9)$ & $0.3(0.1-1.2)$ & $0.1(0.1-0.4)$ & \\
\hline
\end{tabular}

Note: Values for categorical variables are given as number (percentage); values for continuous variables, as mean (standard deviation) or median (interquartile range)

CRIC Chronic Renal Insufficiency Cohort; eGFR estimated glomerular filtration rate; HS high school; PVD peripheral vascular disease

${ }^{a}$ Self-reported 
propensity score matching was assessed using standardized differences for each covariate between the matched groups. The primary analysis used a Cox proportional hazards model stratified by this matched pair sample. In addition, we conducted the following sensitivity analyses: 1) we used Cox proportional hazard models to evaluate the association of prior nephrology contact with each outcome by adding the propensity score in the fully adjusted model as a covariate; 2) for the outcomes of incident CVD and all-cause mortality, we conducted multivariable regression analyses censoring at the time of ESRD; 3) we repeated the main regression analyses censoring at a follow-up time of 3.6 years instead of 6.6 years; and 4) for each outcome, we evaluated the effect of timing of nephrology contact prior to study enrollment (i.e., initial nephrology contact $\geq 1$ year before baseline visit, initial nephrology contact $<1$ year prior to baseline visit, or no prior nephrology contact). All hypothesis tests were two-sided with 0.05 alpha level. Regression models were adjusted for clinical center. The Supremum test was used to evaluate the Cox's models proportionality assumption. ${ }^{25}$ Statistical analyses were conducted using SAS, version 9.3 (Cary, NC).

\section{RESULTS}

\section{Cohort Characteristics}

After exclusion of 73 participants with missing data regarding prior nephrology contact and 11 participants with eGFR < $15 \mathrm{ml} / \mathrm{min} / 1.73 \mathrm{~m}^{2}$ at study entry, we studied a total of 3855 CRIC participants. Their mean (SD) age at baseline was 58 (11) years, $55 \%$ were male, and $42 \%$ were non-Hispanic white (Table 1). The $67 \%$ of participants who reported nephrology contact prior to study entry were more likely to be male and to have health insurance; and had lower eGFR and higher urine protein excretion compared with those who did not report such contact. A comparison of demographic and clinical characteristics between propensity score matched pairs is presented in Table $1 \mathrm{~S}$ (supplementary material).

\section{Baseline Factors Associated with Prior History of Contact with a Nephrologist}

In multivariable logistic regression analyses, increasing age and body mass index (BMI), female sex, Hispanic ethnicity and diabetes were associated with lower odds of prior nephrology contact, while health insurance and high school education or higher were associated with higher odds of such contact. Lower eGFR and higher proteinuria were also associated with significantly higher odds of prior nephrology contact (Table 2).

\section{Continuity of Nephrology Care}

Among the 1019 participants with either eGFR $15-<30 \mathrm{ml} / \mathrm{min} /$ $1.73 \mathrm{~m}^{2}$ or urine protein $\geq 3 \mathrm{~g} /$ day who reported nephrology contact prior to study entry, $662(65 \%)$ reported nephrology contact at least once per year prior to each subsequent follow-up visit (continuity of nephrology care $100 \%), 171$ (17\%) reported nephrology contact prior to most but not all follow-up visits (continuity of nephrology care 51-99\%), and 186 (18\%) reported nephrology contact prior to less than half of the follow-up visits (continuity of nephrology care $\leq 50 \%$ ). In unadjusted analysis, compared with participants with continuity of nephrology care $\leq 50 \%$, those with $100 \%$ continuity of nephrology care were more likely to have annual household income $>\$ 20,000$ (59 vs. $46 \%$ ) and high school education or greater (72 vs. $60 \%$ ); and less likely to be Hispanic (17 vs. $36 \%$ ). The mean (SD) eGFR was $27(7.5) \mathrm{ml} / \mathrm{min} / 1.73 \mathrm{~m}^{2}$ among participants with $100 \%$ continuity of nephrology care, which compared with 31 (11) $\mathrm{ml} / \mathrm{min} / 1.73 \mathrm{~m}^{2}$ among those with $\leq 50 \%$ continuity of nephrology care. The median (IQR) urine protein excretion was $1.3(0.3-4.2) \mathrm{g} / 24 \mathrm{hr}$ for participants with $100 \%$ continuity of nephrology care, and $3.1(0.5-5.1) \mathrm{g} / 24 \mathrm{hr}$ among those with $\leq 50 \%$ continuity of nephrology care (data not shown).

\section{Management of CKD by Prior Nephrology Contact}

Among participants with baseline eGFR $15-<30 \mathrm{ml} / \mathrm{min} /$ $1.73 \mathrm{~m}^{2}$, those who reported prior nephrology contact (compared with those who did not) were more likely to have serum bicarbonate and phosphorus treated and at goal (Table 3). There were no significant differences in achieving the guideline-recommended goals for systolic BP, iPTH or hemoglobin by prior nephrology contact, regardless of eGFR strata.

Table 2 Factors Associated with Contact with a Nephrologist Prior to CRIC Baseline Visit ${ }^{\mathrm{a}}$

\begin{tabular}{lll}
\hline \hline & OR (95 \% CI) & $p$ \\
\hline Age (per 1-year increase) & $0.96(0.95-0.97)$ & $<0.001$ \\
Female (vs. male) & $0.55(0.45-0.67)$ & $<0.001$ \\
Race/ethnicity & Referent group & \\
$\quad$ Non-Hispanic White & $0.32(0.22-0.47)$ & $<0.001$ \\
$\quad$ Hispanic & $0.98(0.79-1.22)$ & 0.87 \\
$\quad$ Non-Hispanic Black & $0.94(0.59-1.50)$ & 0.79 \\
$\quad$ Other & & \\
Educational attainment & Referent group & \\
$\quad$ Less than high school education & $1.34(1.04-1.73)$ & 0.03 \\
$\quad$ High school graduate/Some college & $2.02(1.49-2.74)$ & $<0.001$ \\
$\quad$ College graduate or higher & $1.58(1.12-2.21)$ & 0.009 \\
Health insurance (vs. no & & \\
health insurance) & $0.96(0.79-1.15)$ & 0.63 \\
Smoker $\geq 100$ cig/lifetime (Yes vs. No) & $1.00(1.00-1.01)$ & 0.49 \\
Systolic BP (per 1 mmHg) & $1.01(0.83-1.24)$ & 0.89 \\
Any cardiovascular disease & $0.64(0.52-0.78)$ & $<0.001$ \\
Diabetes & $1.11(0.86-1.42)$ & 0.43 \\
Dyslipidemia & $0.97(0.96-0.99)$ & $<0.001$ \\
Body mass index (per 1 unit increase) & $1.94(1.81-2.09)$ & $<0.001$ \\
eGFR (per 10 unit decrement) & $1.31(1.08-1.61)$ & 0.008 \\
Proteinuria (per 1 unit increase in & & \\
log-urine protein, g/24 h) & & \\
\hline
\end{tabular}

${ }^{a}$ Adjusted for clinical center and all variables listed

OR odds ratio; CI confidence interval 
Table 3 Measures of Management of Chronic Kidney Disease by Prior Contact with a Nephrologist Over Three Ranges of Estimated Glomerula Filtration Rate

\begin{tabular}{|c|c|c|c|c|c|c|}
\hline \multirow[b]{3}{*}{ Variables } & \multirow{2}{*}{\multicolumn{2}{|c|}{$\frac{\text { eGFR } 15-<30 \mathrm{ml} / \mathrm{min} / 1.73 \mathrm{~m}^{2}}{\text { Prior Contact with Nephrologist }}$}} & \multicolumn{2}{|c|}{ eGFR $30-<60 \mathrm{ml} / \mathrm{min} / 1.73 \mathrm{~m}^{2}$} & \multicolumn{2}{|c|}{ eGFR $\geq 60 \mathrm{ml} / \mathrm{min} / 1.73 \mathrm{~m}^{2}$} \\
\hline & & & & & & \\
\hline & Yes $(n=705)$ & No $(n=87)$ & Yes $(n=1597)$ & No $(n=778)$ & Yes $(n=289)$ & No $(n=399)$ \\
\hline Systolic BP, mm Hg & $133(24)$ & $133(22)$ & $128(22)$ & $130(23)$ & $121(18)$ & $122(19)$ \\
\hline At goal $(<130 / 80 \mathrm{~mm} \mathrm{Hg})$ & $48 \%(337 / 705)$ & $45 \%(39 / 87)$ & $57 \%(908 / 1596)$ & $53 \%(410 / 778)$ & $74 \%(213 / 289)$ & $72 \%(289 / 399)$ \\
\hline Treated $^{\mathrm{a}}$ and at goal & $48 \%(324 / 681)$ & $47 \%(39 / 83)$ & $56 \%(848 / 1511)$ & $53 \%(386 / 734)$ & $71 \%(167 / 235)$ & $66 \%(178 / 271)$ \\
\hline Bicarbonate, mEq/L & $23(3)$ & $23(4)$ & $24(3)^{*}$ & $25(3)^{*}$ & $26(3)$ & $26(3)$ \\
\hline At goal ( $\geq 22 \mathrm{mEq} / \mathrm{L})$ & $64 \%(451 / 698)$ & $63 \%(55 / 87)$ & $84 \%(1340 / 1585)$ & $86 \%(669 / 773)$ & $91 \%(262 / 281)$ & $94 \%(376 / 397)$ \\
\hline Treated $^{\mathrm{b}}$ and at goal & $65 \%(26 / 40)^{*}$ & $0^{*}$ & $73 \%(16 / 22)$ & $50 \%(2 / 4)$ & $50 \%(1 / 2)$ & 0 \\
\hline Phosphate, mg/dL & $4.1(1)$ & $4.2(1)$ & $3.7(1)$ & $3.7(1)$ & $3.4(1)^{*}$ & $3.5(1)^{*}$ \\
\hline At goal $(2.7-4.6 \mathrm{mg} / \mathrm{dL})$ & $75 \%(530 / 687)$ & $71 \%(62 / 87)$ & $89 \%(1423 / 1574)$ & $90 \%(697 / 766)$ & - & - \\
\hline Treated $^{\mathrm{c}}$ and at goal & $75 \%(64 / 85)^{*}$ & $46 \%(5 / 11)^{*}$ & $88 \%(87 / 99)$ & $79 \%(33 / 42)$ & - & - \\
\hline iPTH & $109(67-176)$ & $92(61-159)$ & $55(36-84)$ & $49(33-74)$ & $36(28-51)$ & $35(27-47)$ \\
\hline At goal ${ }^{\mathrm{e}}$ (not on treatment) & $24 \%(136 / 564)^{*}$ & $37 \%(28 / 75) *$ & $39 \%(572 / 1458)$ & $43 \%(317 / 736)$ & - & - \\
\hline Treated $^{\mathrm{d}}$ and at goal ${ }^{\mathrm{e}}$ & $53 \%(75 / 141)$ & $58 \%(7 / 12)$ & $48 \%(66 / 139)$ & $62 \%(26 / 42)$ & - & - \\
\hline Hemoglobin, g/dl & $11.7(1.7)$ & $11.5(1.6)$ & $12.7(1.7)^{*}$ & $12.5(1.7)^{*}$ & $13.9(1.6)^{*}$ & $13.4(1.5)^{*}$ \\
\hline $\begin{array}{l}\text { At goal }(11-12 \mathrm{~g} / \mathrm{dl} \text {, } \\
\text { not on treatment) }\end{array}$ & $91 \%(480 / 528)^{*}$ & $85 \%(66 / 78)^{*}$ & $96 \%(1366 / 1422)$ & $95 \%(689 / 729)$ & $97 \%(267 / 275)$ & $98 \%(370 / 377)$ \\
\hline Treated $^{\mathrm{f}}$ and at goal & $57 \%(100 / 177)$ & $67 \%(6 / 9)$ & $49 \%(86 / 175)$ & $39 \%(19 / 49)$ & $21 \%(3 / 14)$ & $41 \%(9 / 22)$ \\
\hline ACEi or ARB use & $67 \%(473 / 702)$ & $74 \%(64 / 87)$ & $76 \%(1199 / 1585)^{*}$ & $71 \%(550 / 771)^{*}$ & $60 \%(173 / 287)^{*}$ & $46 \%(181 / 396)^{*}$ \\
\hline
\end{tabular}

Values are expressed as mean (standard deviation), median (interquartile range), or \% (numerator/denominator). For systolic BP, bicarbonate and phosphate, the "at goal" category includes individuals with and without treatment

ACEi angiotensin converting enzyme inhibitor; ARB angiotensin receptor blocker; BP blood pressure; iPTH intact parathyroid hormone

${ }^{a}$ Use of any antihypertensive medication

${ }^{b}$ Use of sodium bicarbonate, potassium bicarbonate or sodium citrate

${ }^{c}$ Use of any phosphorus binder

${ }^{d}$ Use of a phosphorus binder, active vitamin $D$ formulation or cinacalcet

${ }^{e} i P T H$ (pg/ml) treatment goal based on eGFR: 70-110 (eGFR 15-30); 30-59 (eGFR 30-60)

${ }^{f}$ Use of an erythropoietin stimulating agent or iron

$* p<0.05$

A significant greater proportion of participants with eGFR $\geq$ $30 \mathrm{ml} / \mathrm{min} / 1.73 \mathrm{~m}^{2}$ who reported prior nephrology contact were taking ACEi/ARB (Table 3). Similar patterns of higher ACEi/ARB use by prior nephrology contact were observed across strata of proteinuria ( $>0.3 \mathrm{vs} . \leq 0.3 \mathrm{~g} / 24 \mathrm{hr}$, data not shown).

\section{Influence of Prior Contact with a Nephrologist on Outcomes}

During a median length of follow-up of 6.6 years we observed 816 ESRD events, 846 CVD events (744 prior to ESRD), and 707 deaths (493 prior to ESRD). Participants who reported prior nephrology contact were more likely to experience CKD progression, incident CVD, and death during the follow-up period. The unadjusted event rates (per 100 person-year) in participants with prior nephrology contact compared with those without it were 7.5 vs. 3.5 for CKD progression $(\mathrm{p}<0.001) ; 4.6$ vs. 3.4 for incident CVD $(\mathrm{p}<0.001)$; and 3.3 vs. 2.4 for all-cause death $(\mathrm{p}<0.001)$ (Figure $1 \mathrm{~S}$, supplementary material). However, in Cox proportional hazards models using propensity score matched pairs, prior nephrology contact, compared with no such contact, was not associated with CKD progression (hazard ratio (HR) $1.21 ; \mathrm{p}=0.4$ ), CVD events (HR 1.20; $\mathrm{p}=0.3$ ), or death (HR 0.83; $\mathrm{p}=0.3$ ) (Fig. 1). Results obtained from sensitivity analyses were similar to those from the main analyses. Specifically, using propensity score as a covariate in fully adjusted Cox proportional models did not alter the results (data not shown). In analyses of incident CVD and all-cause mortality where ESRD was treated as a censoring event, the results were also unchanged. Censoring at a shorter follow-up time produced similar results.

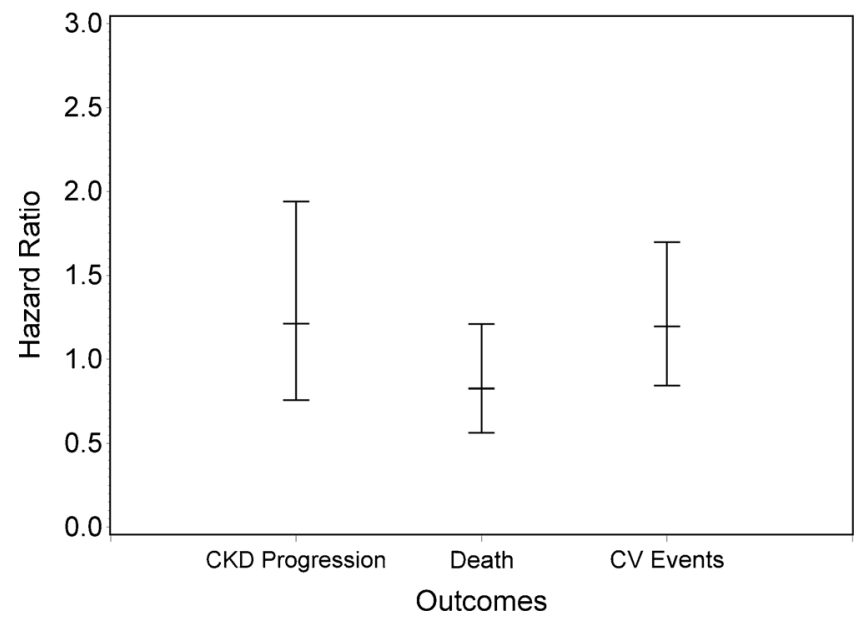

Fig. 1 Hazard ratios and $95 \%$ confidence intervals of chronic kidney disease progression, death and cardiovascular events for individuals with prior contact with a nephrologist (vs. not). Adjusted for clinical center, age, gender, race/ethnicity, educational attainment, smoking, systolic BP, self-reported cardiovascular disease, diabetes, high cholesterol, BMI, baseline eGFR and urine protein excretion. 
Additional sensitivity analyses evaluating timing of prior nephrology contact showed no significant association between prior nephrology contact and the outcomes studied (data not shown). The proportionality assumption of Cox models was met.

\section{DISCUSSION}

In a large and diverse cohort of adults with $\mathrm{CKD}$, one-third reported not seen a nephrologist in the year prior to study entry. We observed significant sociodemographic disparities in prior nephrology contact. Despite accounting for severity of CKD, participants who were older, female, Hispanic, and had lower educational attainment were significantly less likely to report prior nephrology contact. Even though prior nephrology contact was associated with a higher prevalence of treatment for CKD complications and use of ACEi/ARB, there were no consistent differences in achievement of guidelinerecommended goals across the range of eGFR studied. In adjusted analyses, prior nephrology contact was not associated with CKD progression, incident CVD or death.

Similar to our findings, other studies have reported suboptimal prevalence of nephrology care as well as sociodemographic disparities in nephrology care referral. In a single-center retrospective study of 268 stage 3-4 CKD patients, those without nephrology referral were more likely to be $\geq 65$ years, female and non-white. ${ }^{26}$ Furthermore, among over 400 geriatric ambulatory patients with mild to moderate $\mathrm{CKD}$, only $8.8 \%$ were referred to a nephrologist. ${ }^{27}$ In contrast, in a study of 135 patients initiating dialysis, no association was found between age, gender or race and timing of nephrology referral, but private health insurance conferred a higher odds of nephrology referral compared with Medicare. Factors that might influence primary care providers' $(\mathrm{PCP})$ referral of patients with $\mathrm{CKD}$ to nephrologists are not well understood. Although specific characteristics of PCP does not appear to play a role ${ }^{28}$ insufficient awareness of guidelines recommendations for CKD management and nephrology referral has been identified as a potential explanation for low referral rates. ${ }^{26,29}$ In addition, it is possible that certain patient-level factors play an important role including lack of initiative to schedule a nephrology appointment or adherence to scheduled appointments.

Given that we did not observe a difference in CKD treatment goals achievement, it is not surprising that we failed to find a significant association between prior nephrology contact and outcomes. Similar to our findings, the Kidney Early Evaluation Program reported no significant independent association between mortality and nephrology care in participants with eGFR $<60 \mathrm{ml} / \mathrm{min} / 1.73 \mathrm{~m}^{2} .{ }^{23}$ Moreover, a large U.S. study reported no substantial improvement in 1-year survival rates after dialysis initiation despite a consistent trend toward increased use of nephrology consultation in older patients approaching ESRD. ${ }^{30}$ In contrast, three small studies conducted outside the U.S. found nephrology care to be associated with better kidney function preservation. ${ }^{12-14}$ In two separate studies of U.S. Veterans with CKD, nephrology care was associated with a reduced risk of death, and regular frequent visits were associated with the lowest risk. ${ }^{10,11} \mathrm{~A}$ potential explanation for these heterogeneous findings is that patients with severe terminal medical conditions (e.g. active cancer, advanced liver cirrhosis) were excluded from the CRIC Study. These types of patients have shorter lifespans and high mortality, and are generally not referred for nephrology consultation in clinical practice. In prior studies, such patients would be captured in the non-nephrology care comparison group, which would increase the likelihood of observing lower mortality in patients receiving nephrology care. Hence, the failure to include these severely ill patients in the CRIC Study may have resulted in a relatively lower mortality rate in our nonnephrology care group, and may have biased against an observed mortality benefit from nephrology care.

Although CRIC participants with prior nephrology care had a higher use of ACEi/ARB, they did not have consistently higher attainment of guideline-recommended goals. While a few existing studies have noted that adherence to clinical guidelines is suboptimal overall, ${ }^{31,32}$ nephrology care has been generally associated with better control of hypertension, anemia, and calcium-phosphorus metabolism. ${ }^{12-14,33-35}$ However, others have not observed a benefit of nephrology care on these parameters and have found such care to vary substantially among nephrologists. ${ }^{11,36}$ Several reasons may account for our observations. First, the overall achievement of guideline-recommended goals was high in the CRIC Study, and therefore significant differences between patients with and without nephrology care may be difficult to appreciate. Second, patient-centered factors such as access to healthcare services (e.g., medication co-payments, transportation) likely impact these outcomes, but are not available in our data. Lastly, despite stratifying by eGFR, other factors reflecting disease severity (e.g., difficult to control hypertension) that impact our outcomes may be worse in the referred population and introduce selection bias into our analyses.

Strengths of this study include the large sample size and the racial/ethnic diversity of the CRIC Study, as well as the availability of a wide range of clinical measures. However, in addition to the issues discussed above, our study has limitations. First, although we conducted propensity score matched analyses to attenuate confounding by indication bias (i.e., referral to a nephrologist due to worse kidney function and hematologic/metabolic parameters), residual confounding might remain. For example, we were unable to adjust for the number of nephrology visits prior to study enrollment. Second, it is possible that nephrology care ascertainment with a self-administered questionnaire, and not confirmed by insurance claims or other means, led to recall bias and misclassification of the exposure. Lastly, the ascertainment of nephrologist care by presence or absence of clinical visits is only a crude marker of the patient-physician interaction; data regarding other potentially significant variables including visit length 
and patient satisfaction were not collected. Moreover, we did not have enough information at the baseline visit regarding medical care provided by PCP.

In summary, one-third of CRIC participants reported not seen a nephrologist prior to enrollment, and this prior contact was subject to age, sex, and ethnic-related disparities. While prior nephrology care was associated with higher ACEi/ARB use, there was no significant association between nephrology care and long-term outcomes. Further observational studies with longer follow-up time as well as qualitative research studies that examine nephrologist-patient clinic visit interactions in greater detail would be important to better determine the relevance of nephrology care in earlier stages of CKD.

Acknowledgements: Funders: Funding for the CRIC Study was obtained under cooperative agreements from the National Institute of Diabetes and Digestive and Kidney Diseases (UO1DK060990, U01DK060984, U01DK061022, U01DK061021, UO1DK061028, U01DK060980, U01DK060963, and UO1DK060902). In addition, this work was supported in part by: the Perelman School of Medicine at the University of Pennsylvania Clinical and Translational Science Award NIH/NCATS UL1TR000003, Johns Hopkins University UL1 TR000424, University of Maryland GCRC MO1 RR-16500, Clinical and Translational Science Collaborative of Cleveland, UL1TR000439 from the National Center for Advancing Translational Sciences (NCATS) component of the National Institutes of Health and NIH roadmap for Medical Research, Michigan Institute for Clinical and Health Research (MICHR) UL1TR000433, University of Illinois at Chicago CTSA UL1RR029879, Tulane University Translational Research in Hypertension and Renal Biology P3OGM103337, Kaiser Permanente NIH/ NCRR UCSF-CTSI UL1 RR-024131. A.C.R is funded by the National Institutes of Diabetes and Digestive and Kidney Diseases 1K23DK094829-01 Award.

Prior Presentation: This work was presented in the abstract form at the 2012 American Society of Nephrology Meeting in San Diego, CA.

Conflict of Interest: The authors declare that they do not have a conflict of interest

Corresponding Author: Ana C. Ricardo, MD, MPH; Section of Nephrology University of Illinois at Chicago, 820 South Wood Street, Room 470 (M/C 793), Chicago, IL 60612-7315, USA (e-mail: aricar2@uic.edu).

\section{REFERENCES}

1. K/DOQI clinical practice guidelines for chronic kidney disease: evaluation, classification, and stratification. Am J Kidney Dis. 2002; 39: S1-266.

2. Kidney Disease: Improving Global Outcomes (KDIGO) CKD Work Group. KDIGO 2012 Clinical Practice Guideline for the Evaluation and Management of Chronic Kidney Disease. Kidney Inter., Suppl. 2013; 3: 1-150. 2013.

3. Smart NA, Titus TT. Outcomes of early versus late nephrology referral in chronic kidney disease: a systematic review. Am J Med. 2011;124:1073-1080.

4. Smart NA, Dieberg G, Ladhani M, Titus T. Early referral to specialist nephrology services for preventing the progression to end-stage kidney disease. Cochrane Database Syst Rev. 2014;6, CD007333.

5. Avorn J, Bohn RL, Levy E, et al. Nephrologist care and mortality in patients with chronic renal insufficiency. Arch Intern Med. 2002; 162:2002-2006.

6. Chan MR, Dall AT, Fletcher KE, Lu N, Trivedi H. Outcomes in patients with chronic kidney disease referred late to nephrologists: a meta-analysis. Am J Med. 2007;120:1063-1070.

7. Khan SS, Xue JL, Kazmi WH, et al. Does predialysis nephrology care influence patient survival after initiation of dialysis? Kidney Int. 2005;67:1038-1046.
8. Kinchen KS, Sadler J, Fink N, et al. The timing of specialist evaluation in chronic kidney disease and mortality. Ann Intern Med. 2002;137:479-486.

9. Stack AG. Impact of timing of nephrology referral and pre-ESRD care on mortality risk among new ESRD patients in the United States. Am J Kidney Dis. 2003;41:310-318.

10. Tseng CL, Kern EF, Miller DR, et al. Survival benefit of nephrologic care in patients with diabetes mellitus and chronic kidney disease. Arch Intern Med. 2008;168:55-62.

11. Orlando LA, Owen WF, Matchar DB. Relationship between nephrologist care and progression of chronic kidney disease. N C Med J. 2007;68:9-16.

12. Jones C, Roderick $\mathbf{P}$, Harris $\mathbf{S}$, Rogerson $\mathbf{M}$. Decline in kidney function before and after nephrology referral and the effect on survival in moderate to advanced chronic kidney disease. Nephrol Dial Transplant. 2006;21:2133-2143.

13. Martinez-Ramirez HR, Jalomo-Martinez B, Cortes-Sanabria L, et al. Renal function preservation in type 2 diabetes mellitus patients with early nephropathy: a comparative prospective cohort study between primary health care doctors and a nephrologist. Am J Kidney Dis. 2006;47:78-87.

14. Chen SC, Chang JM, Chou MC, et al. Slowing renal function decline in chronic kidney disease patients after nephrology referral. Nephrology (Carlton). 2008; 13:730-736.

15. Feldman HI, Appel LJ, Chertow GM, et al. The chronic renal insufficiency cohort (CRIC) study: design and methods. J Am Soc Nephrol. 2003; 14:S148-S153.

16. Lash JP, Go AS, Appel LJ, et al. Chronic renal insufficiency cohort (CRIC) study: baseline characteristics and associations with kidney function. Clin J Am Soc Nephrol. 2009;4:1302-1311.

17. Fischer MJ, Go AS, Lora CM, et al. CKD in Hispanics: baseline characteristics from the CRIC (Chronic Renal Insufficiency Cohort) and Hispanic-CRIC studies. Am J Kidney Dis. 2011;58:214-227.

18. K/DOQI clinical practice guidelines for bone metabolism and disease in chronic kidney disease. Am J Kidney Dis. 2003; 42: S1-201.

19. K/DOQI clinical practice guidelines on hypertension and antihypertensive agents in chronic kidney disease. Am J Kidney Dis. 2004; 43: S1-290.

20. II. Clinical practice guidelines and clinical practice recommendations for anemia in chronic kidney disease in adults. Am J Kidney Dis. 2006; 47: S16-S85.

21. Anderson AH, Yang W, Hsu CY, et al. Estimating GFR among participants in the Chronic Renal Insufficiency Cohort (CRIC) study. Am J Kidney Dis. 2012;60:250-261.

22. Campbell KH, Smith SG, Hemmerich J, et al. Patient and provider determinants of nephrology referral in older adults with severe chronic kidney disease: a survey of provider decision making. BMC Nephrol. 2011;12:47.

23. Saab G, Chen SC, Li S, et al. Association of physician care with mortality in Kidney Early Evaluation Program (KEEP) participants. Am J Kidney Dis. 2012;59:S34-S39.

24. Wauters JP, Lameire N, Davison A, Ritz E. Why patients with progressing kidney disease are referred late to the nephrologist: on causes and proposals for improvement. Nephrol Dial Transplant. 2005;20:490-496.

25. Wei LJ. Testing goodness of fit for proportional hazards model with censored observations. J Am Stat Assoc. 1984;79:649-652.

26. Navaneethan SD, Kandula P, Jeevanantham V, Nally JV Jr, Liebman SE. Referral patterns of primary care physicians for chronic kidney disease in general population and geriatric patients. Clin Nephrol. 2010;73:260267.

27. Boudville $\mathbf{N}$, Muthucumarana $\mathbf{K}$, Inderjeeth $\mathbf{C}$. Limited referral to nephrologists from a tertiary geriatric outpatient clinic despite a high prevalence of chronic kidney disease and anaemia. BMC Geriatr. 2012;12:43.

28. Montgomery AJ, McGee HM, Shannon W, Donohoe J. Factors influencing general practitioner referral of patients developing end-stage renal failure: a standardised case-analysis study. BMC Health Serv Res. 2006;6: 114 .

29. Boulware LE, Troll MU, Jaar BG, Myers DI, Powe NR. Identification and referral of patients with progressive CKD: a national study. Am J Kidney Dis. 2006; $48: 192-204$

30. Winkelmayer WC, Liu J, Chertow GM, Tamura MK. Predialysis nephrology care of older patients approaching end-stage renal disease. Arch Intern Med. 2011;171:1371-1378.

31. Hoy T, Fisher M, Barber B, Borker R, Stolshek B, Goodman W. Adherence to K/DOQI practice guidelines for bone metabolism and disease. Am J Manage Care. 2007;13:620-625.

32. Cleveland DR, Jindal KK, Hirsch DJ, Kiberd BA. Quality of prereferral care in patients with chronic renal insufficiency. Am J Kidney Dis. 2002:40:30-36. 
33. Patwardhan MB, Samsa GP, Matchar DB, Haley WE. Advanced chronic kidney disease practice patterns among nephrologists and non-nephrologists: a database analysis. Clin J Am Soc Nephrol. 2007;2:277-283.

34. Philipneri MD, Rocca Rey LA, Schnitzler MA, et al. Delivery patterns of recommended chronic kidney disease care in clinical practice: administrative claims-based analysis and systematic literature review. Clin Exp Nephrol. 2008;12:41-52.
35. Winkelmayer WC, Levin R, Avorn J. The nephrologist's role in the management of calcium-phosphorus metabolism in patients with chronic kidney disease. Kidney Int. 2003;63:1836-1842.

36. Curtis BM, Barrett BJ, Djurdjev O, Singer J, Levin A. Evaluation and treatment of CKD patients before and at their first nephrologist encounter in Canada. Am $J$ Kidney Dis. 2007;50:733-742. 